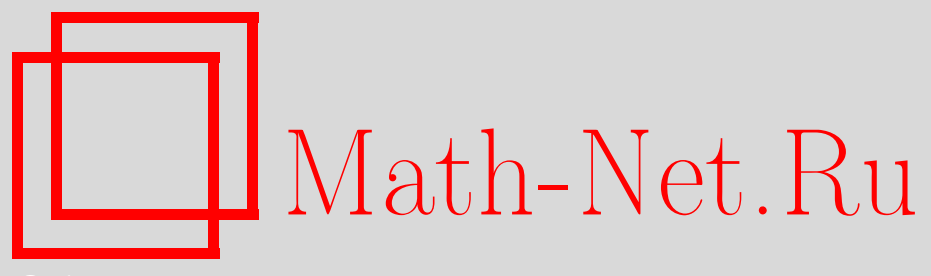

А. П. Шашкин, Закон повторного логарифма для ассоциированного случайного поля, УМН, 2006, том 61, выпуск 2, 173-174

DOI: https://doi.org/10.4213/rm1729

Использование Общероссийского математического портала Math-Net.Ru подразумевает, что вы прочитали и согласны с пользовательским соглашением http://www . mathnet.ru/rus/agreement

Параметры загрузки:

IP : 34.229 .45 .116

26 апреля 2023 г., 14:48:42

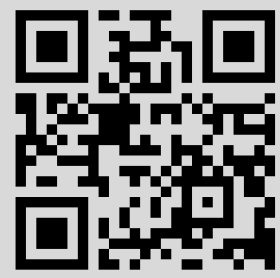




\section{Закон повторного логарифма для ассоциированного случайного поля}

\section{А. П. Шашкин}

Заметка посвящена установлению закона повторного логарифма для нормированных частичных сумм случайного поля, обладающего свойством ассоциированности. Напомним, что случайное поле $X=\left\{X_{j}, j \in \mathbb{Z}^{d}\right\}$ называется ассоциированным [1], если

$$
\operatorname{cov}\left(f\left(X_{i_{1}}, \ldots, X_{i_{n}}\right), g\left(X_{i_{1}}, \ldots, X_{i_{n}}\right)\right) \geqslant 0
$$

для любого $n \in \mathbb{N}$, произвольного набора попарно различных индексов $\left\{i_{1}, \ldots, i_{n}\right\} \subset$ $\mathbb{Z}^{d}$ и любых ограниченных покоординатно неубывающих функций $f, g: \mathbb{R}^{n} \rightarrow \mathbb{R}$. Ассоциированность и родственные ей понятия важны в статистической физике, теории надежности, теории случайных мер. Независимые случайные величины и неубывающие функции от них ассоциированны; известны достаточные условия ассоциированности гауссовских, устойчивых, марковских процессов. В 1980-1990 гг. для ассоциированных случайных полей получены многие классические предельные теоремы теории вероятностей: центральная предельная теорема и ее уточнения, принципы инвариантности, законы больших чисел и др. (см. [2], [3] и там же ссылки). При этом оказалось, что выполнение этих теорем, как правило, обеспечивается моментными ограничениями и определенным поведением ковариационной функции (достаточно быстрым убыванием ковариаций с ростом расстояния между элементами поля). А именно, предполагается, что

$$
u_{1}<\infty \text { и } u_{r} \rightarrow 0 \text { при } r \rightarrow \infty \text {, где } u_{r}=\sup _{j \in \mathbb{Z}^{d}} \sum_{k \in \mathbb{Z}^{d}:|k-j| \geqslant r} \operatorname{cov}\left(X_{j}, X_{k}\right), r \in \mathbb{N} \text {, }
$$

- коэффициент Кокса-Гримметта. Здесь $|j|=\max _{i=1, \ldots, d}\left|j_{i}\right|$ для $j \in \mathbb{Z}^{d}$. Может требоваться также определенная скорость сходимости $u_{r}$ к нулю.

В работах [4] и [5] доказан закон повторного логарифма (обычный и функциональный соответственно) для строго стационарной ассоциированной случайной последовательности в ситуации, когда имеется абсолютный момент порядка выше второго и $u_{r} \rightarrow 0$ (при $r \rightarrow \infty$ ) достаточно быстро. В работе [2] установлен функциональный закон повторного логарифма для ассоциированного случайного поля, из которого вытекает обычный закон повторного логарифма для сумм случайных величин, берущихся по кубам $U_{n}=((0, n] \times \cdots \times(0, n]) \cap \mathbb{Z}^{d}, n \in \mathbb{N}$. Известно, что множество почти наверное предельных точек нормированных должным образом сумм элементов случайного поля зависит от того, по какой базе берутся пределы (см. [6]). В данной работе изучается предельное поведение нормированных сумм по параллелепипедам произвольной формы, стороны которых стремятся к бесконечности.

Назовем целочисленным параллелепипедом множество $(a, b]=\left(\left(a_{1}, b_{1}\right] \times \cdots \times\right.$ $\left.\left(a_{d}, b_{d}\right]\right) \cap \mathbb{Z}^{d}$, здесь $a_{i}, b_{i} \in \mathbb{Z}^{d}$ и $a_{i}<b_{i}$ при $i=1, \ldots, d$. Положим $S(U)=\sum_{j \in U} X_{j}$, где $U$ - целочисленный параллелепипед, и $S_{n}=S((0, n])$, если $n \in \mathbb{N}^{d}$. Для индекса $n \in \mathbb{N}^{d}$ и числа $c \in \mathbb{N}, c>1$, будем обозначать $[n]=n_{1} \cdots n_{d}, c^{n}=\left(c^{n_{1}}, \ldots, c^{n_{d}}\right) \in \mathbb{N}^{d}$, $c^{n-1}=\left(c^{n_{1}-1}, \ldots, c^{n_{d}-1}\right) \in \mathbb{N}^{d}$. Далее $\log x:=\ln (\max \{x, e\}), x>0$, а запись $n \rightarrow \infty$, где $n \in \mathbb{N}^{d}$, означает, что $n_{1} \rightarrow \infty, \ldots, n_{d} \rightarrow \infty$.

Теорема. Пусть стационарное в широком смысле центрированное случайное поле $X$ ассоииированно и таково, что $\sup _{j \in \mathbb{Z}^{d}} \mathrm{E}\left|X_{j}\right|^{2+\delta}<\infty$ для некоторого $\delta>0$. Предположим, что условие (1) выполнено и существует $\lambda>0$ такое, что $u_{r}=$ $O\left(r^{-\lambda}\right)$ при $r \rightarrow \infty$. Тогда, с вероятностью единица,

$$
\limsup _{n \rightarrow \infty} \frac{S_{n}}{\sqrt{2 d[n] \log \log [n]}}=-\liminf _{n \rightarrow \infty} \frac{S_{n}}{\sqrt{2 d[n] \log \log [n]}}=\sigma,
$$

əде $\sigma^{2}=\sum_{j \in \mathbb{Z}^{d}} \operatorname{cov}\left(X_{0}, X_{j}\right)$.

Работа выполнена при поддержке РФФИ (грант № 03-01-00724) и Программы поддержки ведущих научных школ (грант НШ-1758.2003.1). 
Наметим ход доказательства теоремы. Можно считать, что $\sigma>0$, так как иначе утверждение тривиально. Верхняя оценка получается путем стандартного рассуждения с использованием максимального неравенства для ассоциированных полей из работы [7] и результатов о точности нормального приближения для ассоциированного случайного поля (см., например, [3]). Доказательство нижней оценки более сложно. Для $k \in \mathbb{N}^{d}$, достаточно большого $c \in \mathbb{N}$ и малого $\varepsilon>0$ введем целочисленные параллелепипеды $B_{k}=\left(c^{k-1}, c^{k}\right]$ и события $E_{k}=\left\{S\left(B_{k}\right) \geqslant(1-\varepsilon) \sqrt{2 d \mathrm{D} S\left(B_{k}\right) \log \log \left[c^{k}\right]}\right\}$, $k \in G_{\rho}$; здесь $G_{\rho}=\left\{k \in \mathbb{N}^{d}: \min _{i} k_{i} \geqslant\left(k_{1}+\cdots+k_{d}\right)^{\rho}\right\}$ для некоторого $\rho \in(0,1)$. Задача сводится к проверке того, что с вероятностью единица происходит бесконечное число событий $E_{k}$. В свою очередь, согласно классической лемме Эрдёша и Реньи, последнее вытекает из соотношения

$$
\liminf _{n \rightarrow \infty} \frac{\sum_{i, j: 1 \leqslant i, j \leqslant n}\left(\mathrm{P}\left(A_{i} A_{j}\right)-\mathrm{P}\left(A_{i}\right) \mathrm{P}\left(A_{j}\right)\right)}{\left(\sum_{i=1}^{n} \mathrm{P}\left(A_{i}\right)\right)^{2}}=0,
$$

где $\left\{A_{i}\right\}_{i \in \mathbb{N}}-$ события $\left\{E_{k}\right\}_{k \in G_{\rho}}$, перенумерованные по возрастанию величины $k_{1}+\cdots+k_{d}$ (индексы $k \in \mathbb{N}^{d}$, для которых эти величины равны, упорядочиваются, например, в лексикографическом порядке).

Для ассоциированного случайного вектора $(\xi, \eta)$ и липшицевых функций $f, g: \mathbb{R} \rightarrow \mathbb{R}$ справедлива оценка

$$
|\operatorname{cov}(f(\xi), g(\eta))| \leqslant \operatorname{Lip}(f) \operatorname{Lip}(g) \operatorname{cov}(\xi, \eta),
$$

если правая часть определена (см. [8]). С помощью этого утверждения можно проверить соотношение $(2)$, взяв $\xi=S\left(B_{k}\right) / \sqrt{\mathrm{D} S\left(B_{k}\right)}$ и $\eta=S\left(B_{l}\right) / \sqrt{\mathrm{D} S\left(B_{l}\right)}, k, l \in G_{\rho}$. Однако в (3) участвуют липшицевы функции, тогда как в числителе (2) находятся ковариации индикаторов. Поэтому эти индикаторы необходимо сгладить, рассматривая вместо индикатора $\mathbb{I}\{x \geqslant a\}$ функцию $f_{\gamma, a}(x)$, равную нулю при $x \leqslant a$, единице при $x \geqslant a+\gamma$ и линейную на отрезке $[a, a+\gamma]$. Здесь $a=(1-\varepsilon) \sqrt{2 d \log \log \left[c^{k}\right]}$ для $k \in G_{\rho}$, а $\gamma$ - параметр, выбираемый специальным образом для данного индекса $k$. Возникающая в результате случайная погрешность оценивается с помощью теоремы о точности нормального приближения для нормированных сумм, а сумма ковариаций липшицевых функций - посредством (3). Важную роль при этом играет следующая лемма, имеющая самостоятельный интерес. Положим $Z_{k}=S\left(B_{k}\right) / \sqrt{\left|B_{k}\right|}, k \in G_{\rho}$.

Лемма. Двойная сумма $I=\sum_{k, l \in G_{\rho}, k \neq l} \operatorname{cov}\left(Z_{k}, Z_{l}\right)$ конечна.

Отметим, что для получения этой леммы необходимо по-разному оценивать ковариации сумм для "близких" индексов $k$ и $l$ (т.е. таких пар, что $\sum_{i=1}^{d}\left(k_{i}-l_{i}\right)=0$ ) и "далеких" (всех остальных пар). В первом случае следует воспользоваться результатами о поведении дисперсий сумм элементов стационарного поля по растущим параллелепипедам. Именно, из условия (1) и того, что $\sigma>0$, следует оценка $\left.\left|\sigma^{2}-\right| B_{k}\right|^{-1} \mathrm{D} S\left(B_{k}\right) \mid=O\left(c^{-\lambda\left(k_{1}+\cdots+k_{d}\right)^{\rho} /(1+\lambda)}\right), k \rightarrow \infty$. Во втором случае используется следующая из (1) и условия на скорость убывания $u_{r}$ оценка $\operatorname{cov}\left(S\left(B_{k}\right), S\left(B_{l}\right)\right) \leqslant$ $c_{0} \min \left\{\left|B_{k}\right|,\left|B_{l}\right|\right\}\left(\operatorname{dist}\left(B_{k}, B_{l}\right)\right)^{-\lambda}$, здесь $c_{0} \geqslant 0$ не зависит от $k$ и $l$, a $\operatorname{dist}(\cdot, \cdot)-$ расстояние в $\mathbb{Z}^{d}$, отвечающее норме $|\cdot|$.

Автор благодарит профессора А. В. Булинского за внимание к работе.

\section{Список литературы}

[1] J.D. Esary, F. Proschan, D.W. Walkup, Ann. Math. Statist., 38 (1967), 1466-1474. [2] А. В. Булинский, Фундам. и прикл. матем., 1:3 (1995), 623-639. [3] А. V. Bulinski, С. Suquet, Statist. Probab. Lett., 54:2 (2001), 215-226. [4] H. Yu, Acta Math. Sinica, 29:4 (1986), 507-511. [5] A.R. Dabrowski, H. Dehling, Stochastic Process. Appl., 30:2 (1988), 277-289. [6] M. J. Wichura, Ann. Probab., 2 (1974), 202-230. [7] A. V. Bulinski, M. S. Keane, J. Math. Sci., 81:5 (1996), 2905-2911. [8] А. В. Булинский, Э. Шабанович, Фундам. и прикл. матем., 4:2 (1998), 479-492.

А. П. Шашкин (А. P. Shashkin)

Московский государственный университет им. М. В. Ломоносова

E-mail: shashkin@mech.math.msu.su
Представлено А. В. Булинским Принято редколлегией 02.02 .2006 